Mathematical Research Letters 5, 629-635 (1998)

\title{
FOURIER TRANSFORM OF EXPONENTIAL FUNCTIONS AND LEGENDRE TRANSFORM
}

\author{
Jaeyoung Chung, Dohan Kim and Sung Ki Kim
}

\begin{abstract}
We will prove that if $f$ is a polynomial of even degree then the Fourier transform $\mathcal{F}\left(e^{-f}\right)(\xi)$ can be estimated by $e^{-\epsilon f^{*}(\xi)}$ where $f^{*}(\xi)$ is the Legendre transform of $f$ defined by $f^{*}(\xi)=\sup _{x}(x \xi-f(x))$. This result was previously proved by $\mathrm{H}$. Kang $[\mathrm{K}]$ for a case of a convex polynomial which is a finite sum of monomials of even order with positive coefficients.

Our result is the most general one for the polynomial $f(x)$ since the convexity condition is not imposed and $e^{-f(x)}$ belongs to the space $L^{1}$ if and only if $f(x)$ is a polynomial of even degree with the coefficient of the highest degree $a_{2 m}>0$. Also, we will make a more precise estimate of constants.
\end{abstract}

\section{Introduction}

For the estimate of Fourier transform of exponential functions whose exponent is an even convex polynomial with positive coefficients H. Kang shows the following theorem in $[\mathrm{K}]$.

Theorem 1.1. Let $f(x)$ be an even convex polynomial with positive coefficients of the form $f(x)=\sum_{k=1}^{m} a_{k} x^{2 k}, a_{k} \geq 0, k=1, \ldots, m-1$, and $a_{m}>0$. Then the Fourier transform

$$
\mathcal{F}\left(e^{-f}\right)(\xi)=\int_{-\infty}^{\infty} e^{i x \xi} e^{-f(x)} d x
$$

of $e^{-f(x)}$ can be estimated by $e^{-\epsilon f^{*}(\xi)}$, in other words,

$$
\left|\mathcal{F}\left(e^{-f}\right)(\xi)\right| \leq C f^{-1}(1) e^{-\epsilon f^{*}(\xi)},
$$

where the positive constants $C$ and $\epsilon$ depend only on the degree $m$.

Here the Fourier transform $\mathcal{F} \varphi(\xi)$ is defined by $\mathcal{F} \varphi(\xi)=\int e^{i x \xi} \varphi(x) d x$ and the Legendre transform $\psi^{*}$ is defined by $\psi^{*}(\xi)=\sup _{x}(x \xi-\psi(x))$.

In this paper we generalize the above Theorem 1.1 to any polynomial of even degree. This result is the most general for the polynomials $f(x)$, since $e^{-f(x)}$

Received January 23, 1998.

1991 Mathematics Subject Clssification. 42A38, 26A51 
belongs to the space $L^{1}$ if and only if $f(x)$ is a polynomial of even degree with $a_{2 m}>0$. Also, we make a more precise estimate of constants.

Our result shows the clear relation between Fourier transform and Legendre transform, and is related to the duality of phase as E. M. Stein calls in [S, p358] as follows: The Fourier transform of $e^{i \Phi(x)} a(x)$ is essentially of the form $e^{-i \Phi^{*}(\xi)} b(\xi)$, where $a(x)$ is non-oscillatory but regularly varying.

In Section 2 we prove the main theorem and obtain a similar estimate for the general entire convex functions $\psi(x)$ in the Gelfand-Shilov spaces of type $W$. In Section 3 we give a simpler proof of Kang's Theorem 1.1 as an application of the method of proof of the main theorem.

\section{Main Theorem}

In order to define the Legendre transform of polynomials of even degree which may not be convex we need the following definition.

Definition 2.1. Let $M(x)$ be a convex function on $\mathbb{R}$ possibly except for a bounded interval such that $M^{\prime}(\infty)=\infty, M^{\prime}(-\infty)=-\infty$. Then the Legendre transform $M^{*}(\xi)$ of $M(x)$ is defined by

$$
M^{*}(\xi)=\sup _{x}(x \xi-M(x))
$$

It is easy to see that $M^{*}(\xi)$ is well defined for all $\xi \in \mathbb{R}$ and is a convex function which is the same as the Legendre transform of the convex minorant of $M(x)$. We refer to Arnold [A], Gelfand-Shilov [GS] and Hörmander [H1] for more details on the properties of the Legendre transform of convex functions.

In particular, if $M(x)$ is a convex even function such that $M(0)=M^{\prime}(0)=0$ then it is well known that the Legendre transform $M^{*}(\xi)$ of $M$ is given by

$$
M^{*}(\xi)=\int_{0}^{\xi} M^{\prime-1}(t) d t
$$

where $M^{\prime-1}(t)$ is the inverse function of $M^{\prime}(t)$. See [GS, K].

We are now in a position to state and prove the main theorem in this paper.

Theorem 2.2. Let $f(x)=\sum_{k=0}^{2 m} a_{k} x^{k}, a_{2 m}>0$, be a polynomial of even degree. Then there exist positive constants $\epsilon$ and $C$ such that

$$
\left|\mathcal{F}\left(e^{-f}\right)(\xi)\right|=\left|\int_{-\infty}^{\infty} e^{i x \xi-f(x)} d x\right| \leq C e^{-\epsilon f^{*}(\xi)}
$$

where $\epsilon$ depends only on the degree $m$.

For the proof we need the following two lemmas. 
Lemma 2.3. Let $f(x)=\sum_{k=0}^{2 m} a_{k} x^{k}, a_{2 m}>0$. Then there exist positive constants $h$ and $C$ such that

$$
\Re f(x+i y) \geq \frac{1}{2} f(x)-h f(y)-C,
$$

where $h$ depends only on the degree $m$.

Proof. Without loss of generality we may assume that $f(x)$ is monic. Then

$$
\begin{aligned}
\Re f(x+i y) & =\sum_{k=0}^{2 m} a_{k} \Re(x+i y)^{k}, \\
& =\sum_{j=0}^{m}\left(\begin{array}{c}
2 m \\
2 j
\end{array}\right)(-1)^{j} x^{2 m-2 j} y^{2 j}+\sum_{k=0}^{2 m-1} \sum_{j=0}^{[k / 2]} a_{k}\left(\begin{array}{c}
k \\
2 j
\end{array}\right)(-1)^{j} x^{k-2 j} y^{2 j} .
\end{aligned}
$$

Thus we can write

$$
\begin{aligned}
& \Re f(x+i y)-\frac{1}{2} f(x)+h f(y) \\
& =\frac{1}{4} x^{2 m}+\sum_{j=1}^{m-1}\left(\begin{array}{c}
2 m \\
2 j
\end{array}\right)(-1)^{j} x^{2 m-2 j} y^{2 j}+\left\{h+(-1)^{m}-\frac{1}{4}\right\} y^{2 m} \\
& +\frac{1}{4} x^{2 m}+\frac{1}{4} y^{2 m}+R(x, y) \\
& =y^{2 m}\left\{\frac{1}{4}\left(\frac{x}{y}\right)^{2 m}+\sum_{j=1}^{m-1}\left(\begin{array}{c}
2 m \\
2 j
\end{array}\right)(-1)^{j}\left(\frac{x}{y}\right)^{2 m-2 j}+h+(-1)^{m}-\frac{1}{4}\right\} \\
& +\frac{1}{4} x^{2 m}+\frac{1}{4} y^{2 m}+R(x, y),
\end{aligned}
$$

where $R(x, y)$ is a polynomial of degree less than $2 m$.

Therefore, if we choose $h$ to be the maximum of the polynomial

$$
p(x)=-\frac{1}{4} x^{2 m}-\sum_{j=1}^{m-1}\left(\begin{array}{c}
2 m \\
2 j
\end{array}\right)(-1)^{j} x^{2 m-2 j}+(-1)^{m+1}+\frac{1}{4},
$$

we have $\Re f(x+i y) \geq \frac{1}{2} f(x)-h f(y)-C$, since $\frac{1}{4} x^{2 m}+\frac{1}{4} y^{2 m}+R(x, y)$ has a minimum $-C$.

Lemma 2.4. Let $f(x)=\sum_{k=0}^{2 m} a_{k} x^{k}, a_{2 m}>0$. Then for any $\epsilon>0$ there exists a positive constant $C$ such that for $0<\epsilon<1$,

$$
f^{*}(\epsilon x) \geq \epsilon^{\alpha /(\alpha-1)} f^{*}(x)-C, \quad 1<\alpha<2 m
$$


and that for $\epsilon>1$,

$$
f^{*}(\epsilon x) \geq \epsilon^{2 \alpha /(2 \alpha-1)} f^{*}(x)-C, \quad m<\alpha .
$$

Proof. Assume that $f(x)$ is monic. For a given $a$, where $2<a<4^{m}$, there exists a positive constant $C$ such that

$$
f(x) \geq a f(x / 2)-C,
$$

which implies that

$$
f(x) \geq a^{r} f\left(x / 2^{r}\right)-C_{r},
$$

for any positive number $r$. Applying the Legendre transform on both sides of (2.7) we have

$$
f^{*}(x) \leq a^{r} f^{*}\left((2 / a)^{r} x\right)+C_{r} .
$$

For given $\epsilon, 0<\epsilon<1$ and $a=2^{\alpha}, 1<\alpha<2 m$ we can choose $r$ so that $(2 / a)^{r}=\epsilon$. Then we obtain

$$
\begin{aligned}
f^{*}(\epsilon x) & \geq 1 / a^{r} f^{*}(x)-C_{r} / a^{r}, \\
& =\epsilon^{\alpha /(\alpha-1)} f^{*}(x)-C_{r} / a^{r},
\end{aligned}
$$

which proves (2.5). Using the above inequality for $a>4^{n}$

$$
f(x) \geq a f(x / 2)-C,
$$

and applying the same method as above we have the inequality $\left(2.5^{\prime}\right)$. Now for the general polynomial let $f(x)=a_{2 m} f_{0}(x)$. Then $f_{0}(x)$ is monic. Thus we have

$$
\begin{aligned}
f^{*}(\epsilon x) & =\left(a^{2 m} f_{0}\right)^{*}(\epsilon x)=a^{2 m} f_{0}^{*}\left(\epsilon x / a_{2 m}\right), \\
& \geq \epsilon^{\alpha /(\alpha-1)} a^{2 m} f_{0}^{*}\left(x / a_{2 m}\right)+C=\epsilon^{\alpha /(\alpha-1)} f^{*}(x)-C .
\end{aligned}
$$

This completes the proof.

Proof of Theorem 2.2. After the change of contour $x \rightarrow x+i y$ it follows from Lemma 2.3 that

$$
\begin{aligned}
\left|\mathcal{F}\left(e^{-f}\right)(\xi)\right| & \leq \int_{-\infty}^{\infty}\left|e^{i(x+i y) \xi-f(x+i y)}\right| d x \\
& \leq e^{-y \xi} \int_{-\infty}^{\infty} e^{-\Re f(x+i y)} d x \\
& \leq C e^{-y \xi+h f(y)}
\end{aligned}
$$


Taking the infimum of $e^{-y \xi+h f(y)}$ for $y \in \mathbb{R}$ and putting $\alpha=3 / 2$ in (2.5) of Lemma 2.4 we obtain

$$
\left|\mathcal{F}\left(e^{-f}\right)(\xi)\right| \leq C e^{-h f^{*}(\xi / h)} \leq C e^{-\frac{1}{h^{2}} f^{*}(\xi)} .
$$

This completes the proof.

Remark 2.5. As we see in the proof of Lemma 2.3 the inequality (2.4) is equivalent to the condition that there exist positive constants $h$ and $C$ such that

$$
\Re f(x+i y) \geq \frac{1}{2} a_{2 m} x^{2 m}-h a_{2 m} y^{2 m}-C,
$$

where $h$ is the same constant as in (2.4). Thus in view of $\left(2.4^{\prime}\right)$ we have the following equivalent, but more transparent formulation

$$
\left|\mathcal{F}\left(e^{-f}\right)(\xi)\right| \leq C \exp \left(-\epsilon a_{2 m}^{-\frac{1}{2 m-1}} \xi^{\frac{2 m}{2 m-1}}\right),
$$

where $\epsilon=(2 m h)^{-\frac{1}{(2 m-1)}}$.

The estimate $\left(2.3^{\prime}\right)$ means that the Fourier transform of $e^{-f(x)}$ can be controlled by the reciprocal of an exponential function of order $2 m /(2 m-1)$ if $f(x)$ is a polynomial of degree $2 m$. Furthermore, the order $2 m /(2 m-1)$ is optimal in view of the uncertainty principle in [H2].

Also we obtain a similar estimate for general entire convex functions $\psi(x)$ in the Gelfand-Shilov spaces of type $W$ as follows.

Theorem 2.6. Let $\psi(z)$ be an entire function such that $\psi(x)$ is a convex function on $\mathbb{R}$ possibly except for a bounded interval. Also, suppose that $\psi^{\prime}(\infty)=$ $\infty, \psi^{\prime}(-\infty)=-\infty$ and $\Re \psi(x+i y) \geq \psi(a x)-\psi(b y)-C$ for some positive constants $a, b$ and $C$. Then we have

$$
\left|\mathcal{F}\left(e^{-\psi}\right)(\xi)\right| \leq C e^{-\psi^{*}(\epsilon \xi)},
$$

for some $\epsilon, C>0$.

Because the proof of Theorem 2.6 is just the same as that of the Main Theorem we omit the proof.

\section{Simpler proof for even convex polynomials with positive coefficients}

Let $f(x)$ be an even convex polynomial of the form

$$
f(x)=\sum_{k=1}^{m} a_{k} x^{2 k}, \quad a_{k} \geq 0, k=1, \ldots, m .
$$

Then we prove the following Propositions which give a much simpler proof of Theorem 1.1 which is the main theorem in Kang $[\mathrm{K}]$. Also, our proof can give a very precise estimate of constants. 
Proposition 3.1. $f^{*}(\epsilon \xi) \geq \epsilon^{2} f^{*}(\xi), \quad 0<\epsilon<1$.

Proof. The inequality $f^{\prime}(\epsilon \xi) \leq \epsilon f^{\prime}(\xi)$ implies $f^{\prime-1}(\epsilon \xi) \geq \epsilon f^{\prime-1}(\xi)$, since $f^{\prime}$ is increasing. Thus by (2.2) and making a change of variable we have

$$
\begin{aligned}
f^{*}(\epsilon \xi) & =\int_{0}^{\epsilon \xi} f^{\prime-1}(t) d t=\epsilon \int_{0}^{\xi} f^{\prime-1}(\epsilon t) d t \\
& \geq \epsilon^{2} \int_{0}^{\xi} f^{\prime-1}(t) d t=\epsilon^{2} f^{*}(\xi) .
\end{aligned}
$$

Proposition 3.2. $\Re f(x+i y) \geq \frac{1}{2} f(x)-2^{4 m^{2}} f(y)$.

Proof. Since

$$
\begin{aligned}
\Re(x+i y)^{2 k} & =\sum_{j=0}^{k}\left(\begin{array}{c}
2 k \\
2 j
\end{array}\right)(-1)^{j} x^{2 k-2 j} y^{2 j} \\
& \geq \frac{1}{2} x^{2 k}+\frac{1}{2} y^{2 k}\left((x / y)^{2 k}-2^{2 m} \sum_{j=0}^{k-1}(x / y)^{2 j}\right) \\
& \geq \frac{1}{2} x^{2 k}-2^{4 m^{2}} y^{2 k},
\end{aligned}
$$

we have

$$
\Re f(x+i y) \geq \sum_{k=1}^{m} a_{k}\left(\frac{1}{2} x^{2 k}-2^{4 m^{2}} y^{2 k}\right)=\frac{1}{2} f(x)-2^{4 m^{2}} f(y) .
$$

Proposition 3.3. $\int_{-\infty}^{\infty} e^{-\frac{1}{2} f(x)} d x \leq \sqrt{2 \pi e} f^{-1}(1)$.

Proof. If $f(1)=1$ then $f(x) \geq\left(x^{2}-1\right)$. Thus we have

$$
\begin{aligned}
\int_{-\infty}^{\infty} e^{-\frac{1}{2} f(x)} d x & =f^{-1}(1) \int_{-\infty}^{\infty} e^{-\frac{1}{2} f\left(\left(f^{-1}(1) x\right)\right)} d x \\
& \leq f^{-1}(1) \int_{-\infty}^{\infty} e^{-\frac{1}{2}\left(x^{2}-1\right)} d x \\
& \leq \sqrt{2 \pi e} f^{-1}(1)
\end{aligned}
$$

Simpler proof of Theorem 1.1. By a contour change and Proposition 3.2 we have

$$
\begin{aligned}
\left|\mathcal{F}\left(e^{-f}\right)(\xi)\right| & \leq \int_{-\infty}^{\infty}\left|e^{i(x+i y) \xi-f(x+i y)}\right| d x \\
& \leq e^{-y \xi} \int_{-\infty}^{\infty} e^{-\frac{1}{2} f(x)+h f(y)} d x .
\end{aligned}
$$


Taking infimum for $y$ and using Proposition 3.1 and 3.3 we obtain

$$
\left|\mathcal{F}\left(e^{-f}\right)(\xi)\right| \leq \sqrt{2 \pi e} f^{-1}(1) e^{-h f^{*}(\xi / h)} \leq \sqrt{2 \pi e} f^{-1}(1) e^{-\frac{1}{h} f^{*}(\xi)},
$$

where $h=2^{4 m^{2}}$. This completes the proof.

\section{Acknowledgements}

We would like to express our gratitude to the referee for the comment in Remark 2.5. J. Chung is partially supported by BSRI and KOSEF 981-0102011-2. D. Kim and S.K. Kim are partially supported by the SNU Research Grant and GARC-KOSEF.

\section{References}

[A] V. Arnold, Mathematical methods of classical mechanics, Springer-Verlag, Berlin-New York, 1978.

[GS] I. M. Gelfand and G. E. Shilov, Generalized functions, Theory of differential equations, vol. 3, Academic Press, New York-London, 1967.

[H1] L. Hörmander, Notion of convexity, Progress in Mathematics, 127, Birkhäuser Boston, Inc., Berlin-New York, 1994.

[H2] _ A uniqueness theorem of Beurling for Fourier transform pairs, Ark. Mat. 29 (1991), 237-240.

[K] H. Kang, On the Fourier transform of $e^{-\psi(x)}$, Studia Math. 98 (1991), 231-234.

[S] E. M. Stein, Harmonic analysis: Real variable methods, orthogonality, and oscillatory integrals, Princeton Mathematical Series, 43. Monographs in Harmonic Analysis, III., Princeton University Press, Princeton, New Jersey, 1993.

Department of Mathematics, Kunsan National University, Kunsan 573-710, Korea E-mail address: jychung@ks.kunsan.ac.kr

Department of Mathematics, Seoul National University, Seoul 151-742, Korea

E-mail address: dhkim@math.snu.ac.kr

Department of Mathematics, Seoul National University, Seoul 151-742, Korea

E-mail address: skkim@math.snu.ac.kr 